\title{
Remodeling of Synaptic Structure in Sensory Cortical Areas In Vivo
}

\author{
Ania K. Majewska, Jessica R. Newton, and Mriganka Sur \\ Department of Brain and Cognitive Sciences, Picower Center for Learning and Memory, Massachusetts Institute of Technology, Cambridge, Massachusetts \\ 02139
}

\begin{abstract}
Although plastic changes are known to occur in developing and adult cortex, it remains unclear whether these changes require remodeling of cortical circuitry whereby synapses are formed and eliminated or whether they rely on changes in the strength of existing synapses. To determine the structural stability of dendritic spines and axon terminals in vivo, we chose two approaches. First, we performed time-lapse two-photon imaging of dendritic spine motility of layer 5 pyramidal neurons in juvenile [postnatal day 28 (P28)] mice in visual, auditory, and somatosensory cortices. We found that there were differences in basal rates of dendritic spine motility of the same neuron type in different cortices, with visual cortex exhibiting the least structural dynamics. Rewiring visual input into the auditory cortex at birth, however, failed to alter dendritic spine motility, suggesting that structural plasticity rates might be intrinsic to the cortical region. Second, we investigated the persistence of both the presynaptic (axon terminals) and postsynaptic (dendritic spine) structures in young adult mice (P40-P61), using chronic in vivo two-photon imaging in different sensory areas. Both terminals and spines were relatively stable, with $>80 \%$ persisting over a 3 week period in all sensory regions. Axon terminals were more stable than dendritic spines. These data suggest that changes in network function during adult learning and memory might occur through changes in the strength and efficacy of existing synapses as well as some remodeling of connectivity through the loss and gain of synapses.
\end{abstract}

Key words: two-photon; dendritic spine; axon terminal; rewired auditory cortex; mouse; development

\section{Introduction}

Sensory input activates neuronal networks in specific areas of the mammalian cortex. The function of these networks relies on the input-specific remodeling of synapses involved in network activity mediated by bidirectional changes in synaptic strength (Heynen et al., 2000; Liu et al., 2004). The structural basis of synaptic plasticity, however, has remained controversial (Harris et al., 2003).

Dendritic spines are the postsynaptic elements of most excitatory cortical synapses (Ramón y Cajal, 1904; DeRobertis and Bennett, 1955; Gray, 1959; Palay, 1965). The unique shape of the spine allows it to act as a biochemical environment (Yuste and Denk, 1995; Koester and Sakmann, 1998; Yuste et al., 2000), and changes in spine shape likely result in changes in synaptic function (Hayashi and Majewska, 2005; Matus, 2005). Spine motility can be regulated by activity both in vitro (Fischer et al., 2000; Korkotian and Segal, 2001) (but see Dunaevsky et al., 1999) and in vivo (Lendvai et al., 2000; Majewska and Sur, 2003; Oray et al., 2004) (but see Konur and Yuste, 2004). Additionally, directed

\footnotetext{
Received 0ct. 18, 2005; revised Jan. 17, 2006; accepted Jan. 21, 2006.

This work was supported by grants from the National Institutes of Health (M.S.). A.K.M. was supported by a Whiteman Science Fellowship and the Burroughs-Wellcome Career Award in Biomedical Sciences. We thank S. Oray, E. Brown, A. Mower, D. Kornack, and C. Louie for help and useful discussions. We are especially grateful to Daniela Tropea for her help.

Correspondence should be addressed to Ania K. Majewska, Department of Neurobiology and Anatomy, University of Rochester Medical School, 601 Elmwood Avenue, Box 603, Rochester, NY 14642. E-mail: ania_majewska@urmc.rochester.edu.

DOI:10.1523/JNEUROSCI.4454-05.2006

Copyright $\odot 2006$ Society for Neuroscience $\quad$ 0270-6474/06/263021-09\$15.00/0
}

changes in spine morphology have been detected following protocols that alter synaptic strength (Lang et al., 2004; Matsuzaki et al., 2004; Okamoto et al., 2004; Zhou et al., 2004).

Changes in synaptic strength and network function may also come about as a result of a physical disassembly of old synapses and formation of new ones. Activity-dependent formation of filopodia has been reported (Maletic-Savatic et al., 1999), as well as bidirectional changes in spine density in dendritic regions in which plasticity has been elicited (Engert and Bonhoeffer, 1999; Nagerl et al., 2004). In support of this, chronic in vivo imaging of dendritic structure in the young adult somatosensory cortex revealed a large turnover of dendritic spines that was sensitive to whisker trimming (Trachtenberg et al., 2002). Chronic imaging of spines performed in the visual cortex, however, showed very stable dendritic spines (Grutzendler et al., 2002). Spine turnover or dynamics have never been examined previously in auditory cortex.

To further understand the role of morphological changes at synapses in vivo and their relationship to sensory input, we performed two-photon imaging experiments in visual, somatosensory, and auditory cortices. In juvenile animals [postnatal day 28 (P28)], dendritic spines located on the apical tuft of layer 5 neurons in auditory and somatosensory cortex exhibited higher levels of motility than those in visual cortex. Rewiring visual input into the auditory cortex did not reduce spine motility, suggesting that morphological plasticity may be regulated by factors intrinsic to the cortical area rather than by sensory input. We also performed chronic imaging experiments to determine the persis- 
tence of both presynaptic and postsynaptic structures. Dendritic spines and axon terminals in superficial cortical layers were relatively stable ( $>80 \%$ could be reidentified over a 3 week imaging period) in young adult animals (P40), with axon terminals showing less turnover than dendritic spines. These data suggest that changes in synapse morphology rather than turnover are more prominent in adult animals for the remodeling of cortical network activity.

\section{Materials and Methods}

Surgical preparation for in vivo imaging. Mice (C57BL/6) expressing green (GFP) or yellow (YFP) fluorescent protein in a subset of cortical layer 5 neurons were used [transgenic line GFP-M and YFP-H (Feng et al., 2000)]. Animals aged 28-61 d were anesthetized using avertin (16 $\mu \mathrm{l} / \mathrm{g}$ body weight; Sigma, St. Louis, MO). The skull was exposed, scrubbed, and cleaned with ethanol. Primary visual, somatosensory, auditory, and motor cortices were identified based on stereotaxic coordinates, and their location was confirmed with tracer injections (see below). All experiments were performed under protocols approved by the Institutional Animal Care and Use Committee of the Massachusetts Institute of Technology and conformed to National Institutes of Health guidelines.

Acute experiments were performed at P28 to determine relative levels of structural remodeling of spines in different cortical areas. For these experiments, the skull was glued to a metal plate, which was then fastened into a metal base that connected directly into the microscope stage for imaging. The skull was thinned using a high-speed dental drill (Fine Science Tools, Foster City, CA) and, in some cases, removed with forceps to create a small $(1 \times 1 \mathrm{~mm})$ craniotomy. The skull was periodically bathed in saline to ensure that the underlying cortex did not experience damage attributable to excessive heat during drilling. During imaging, anesthesia was maintained with periodic injections of avertin. Neurons could be imaged for up to $10 \mathrm{~h}$ after surgery with no apparent structural abnormalities.

Long-term imaging experiments were started at P40. The skull was thinned over the imaging area (Grutzendler et al., 2002) or a large $(2 \times 2$ $\mathrm{mm}$ ) craniotomy was made and sealed with a coverslip to create an optical window (Yuan et al., 1996). A small metal bar was affixed to the skull. The metal bar was then screwed into a plate that connected directly to the microscope stage for stability during imaging. The metal bar also allowed for maintaining head angle and position during different imaging sessions. At the end of the imaging session, animals in which the skull was thinned were sutured and returned to their cage. On the subsequent imaging session (at P47 or P61), animals were reanesthetized and the skull was rethinned. The same area was identified based on the blood vessel pattern and gross dendritic pattern, which remained remarkably stable during this time. All imaging performed over auditory cortex came from thin skull animals because it was too difficult to implant a window in such a lateral position on the animal's head. For consistency in comparing with images taken in auditory cortex, all data presented in this manuscript come from animals whose skull was thinned for imaging. However, data obtained from animals imaged with an optical window showed similar stability $(91.1 \pm 4.4 \%$ stable over 1 week; $n=103$ spines in 3 animals), suggesting that the surgical preparation does not affect spine stability. These animals provide an important point of comparison with previous studies (see Discussion).

At the end of the last imaging session for both acute and long-term experiments, injections of cholera toxin subunit B (List Biologic, Campbell, CA) coupled to Alexa Fluor 594 (Invitrogen, Carlsbad, CA) were made adjacent to imaged areas to facilitate identification of imaged cells and cortical areas after fixation. Mice were transcardially perfused and fixed with paraformaldehyde, and coronal sections were cut to verify the location of imaged cells. In some cases, sections were incubated with mouse anti-synapsin 1 (Chemicon, Temecula, CA) and subsequently with biotinylated anti-mouse antibody (Vector Laboratories, Burlingame, CA). Sections were then mounted in buffer, coverslipped, and sealed. Images were collected using a Fluoview confocal microscope (Olympus Optical, Melville, NY).
Surgical preparation for rewiring visual input to the auditory pathway. We followed surgical procedures that have been described previously (Lyckman et al., 2001). Briefly, mice were anesthetized $1 \mathrm{~d}$ after birth by deep hypothermia. Under microscopic guidance, bilateral lesions of the inferior colliculus were made, inducing retinal axons to innervate the deafferented medial geniculate nucleus (MGN). On completion of the surgery, the skin was sutured, and the pups were revived under a heat lamp. They were then returned to their mothers and reared until P28 or P40 before being used in imaging experiments.

Two-photon imaging. A custom-made two-photon laser scanning microscope (Majewska et al., 2000b) was used for in vivo imaging. The microscope consists of a modified Fluoview confocal scan head (Olympus Optical) and a titanium/sulphur laser providing $100 \mathrm{fs}$ pulses at 80 $\mathrm{MHz}$ at a wavelength of $920 \mathrm{~nm}$ (Tsunami; Spectra-Physics, Menlo Park, CA) pumped by a $10 \mathrm{~W}$ solid-state source (Millenia; Spectra-Physics). Fluorescence was detected using photomultiplier tubes (HC125-02; Hamamatsu, Shizouka, Japan) in whole-field detection mode. The craniotomy over the visual cortex was initially identified under whole-field fluorescence illumination, and areas with superficial dendrites were identified using a $20 \times, 0.95$ numerical aperture lens (IR2; Olympus Optical). Spiny dendrites were further identified under digital zoom (7$10 \times$ ) using two-photon imaging, and spines 50-200 $\mu \mathrm{m}$ below the pial surface were studied. Image acquisition was accomplished using Fluoview software. For motility measurements, $Z$ stacks taken $0.5-1 \mu \mathrm{m}$ apart were acquired every $5 \mathrm{~min}$ for $2 \mathrm{~h}$. For synapse turnover experiments, $Z$ stacks of dendrites and axons were acquired at $\mathrm{P} 40$ and then again at P47 or P60. Dendrites and axons located in layers 1-3 were studied. Although both layer 5 and layer 6 neurons are labeled in the mice used in this study, only layer 5 neurons send a clear apical dendrite close to the pial surface (Zuo et al., 2005); thus, the data presented come from spines on the apical tuft of layer 5 neurons and axons in superficial cortical layers.

Data analysis. Images were exported to Matlab (MathWorks, Natick, MA) in which they were processed using custom-written algorithms for image enhancement and alignment of the time series. For motility measurements (Majewska and Sur, 2003), spines were analyzed on twodimensional projections containing between 5 and 30 individual images; therefore, movements in the $z$ dimension were not analyzed. Spine motility was defined as the average change in length per unit time (micrometers per minute). Lengths were measured from the base of the protrusion to its tip. This method has the advantage of being insensitive to drift in the images over time and therefore does not require precise alignment of the images. No obvious rotational drift was observed. Dendrites remained stable over the time course of imaging, and the $x-y$ distances between dendritic sections located in different $z$ planes were measured periodically to verify lack of rotation. Additionally, motile spines were often located next to stable spines, suggesting a lack of systemic motion artifacts. For turnover measurements, dendritic spines and axon terminals were identified manually in NIH ImageJ by stepping through the $Z$ stack without projection. Axon terminals were identified as finger-like protrusions $(>0.2 \mu \mathrm{m})$ from the axon or bead-like structures along the axon (at least two times the axon diameter). In fixed sections, axon terminals picked out using these morphological criteria corresponded to puncta of synapsin staining (supplemental Fig. 1, available at www. jneurosci.org as supplemental material). The position of spines and terminals were compared on different imaging days. Spines that were farther than $0.5 \mu \mathrm{m}$ laterally from their previous location were considered to be different spines. Values for stable spines were defined as the percentage of the original spine population present on the second day of imaging. Only areas that showed high signal-to-noise ratio in all imaging sessions were considered for analysis. In both acute and long-term experiments, spines were characterized into classes based on their lengths, spine head diameters, and neck diameters (Harris et al., 1992). Values are presented as mean \pm SE for motility measurements and mean \pm SD in turnover experiments to aid in comparison with previous studies. Filopodia were included in the analysis unless stated otherwise. Analysis was performed blind with respect to animal age and sensory cortical area. 


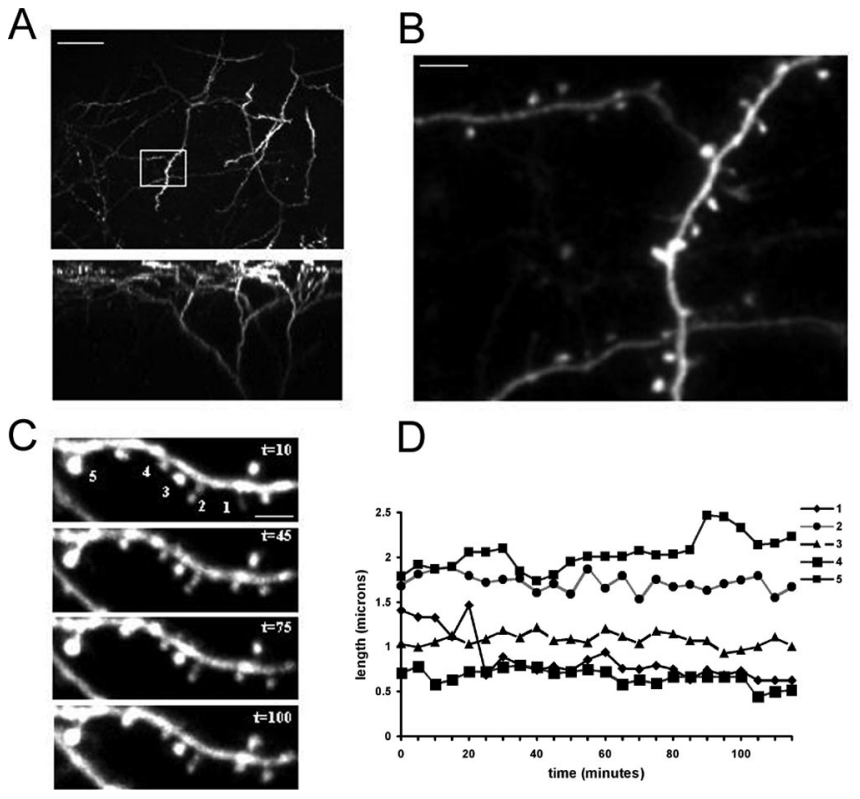

Figure 1. Imaging dendritic spines in vivo in different sensory areas. $\boldsymbol{A}$, Images of the apical tuft of a layer 5 pyramidal neuron in auditory cortex from a P28 animal taken using two-photon microscopy. Top, Collapsed Z stack showing the dendritic arbor (top view). Bottom, Threedimensional reconstruction showing the dendritic arbor from the side. Scale bar, $50 \mu \mathrm{m}$. $\boldsymbol{B}$, Two-photon images of dendritic spines in vivo from dendrite shown in $\boldsymbol{A}$ (boxed area). Scale bar, $5 \mu \mathrm{m}$. C, In vivo image of dendritic section from primary visual cortex of a P28 animal taken at different time points. The time in minutes is indicated in the top right corner of each panel. Scale bar, $5 \mu \mathrm{m}$. D, Graph showing the lengths (from tip to dendrite) over a $2 \mathrm{~h}$ period of five protrusions numbered in $\boldsymbol{A}$. Notice that motile spines such 1 and 5 can be situated next to immotile spines (2-4).

\section{Results}

\section{Imaging spine motility in sensory cortex in vivo}

We studied the motility of dendritic spines in vivo in visual, somatosensory, and auditory cortices of P28 mice. We concentrated our measurements on distal spines located on the apical tuft of layer 5 pyramidal neurons (Fig. $1 A, B$ ). Because this population of spines had been studied previously at this age in primary visual cortex (Majewska and Sur, 2003), we wanted to extend those findings to understand whether basal spine motility is linked to sensory input, cortical area, or a sensory critical period. For consistency with previous studies, spine motility was quantified as the absolute change in length (from dendrite to the tip of the protrusion) per unit time (Lendvai et al., 2000; Majewska and Sur, 2003). Visual, somatosensory, and auditory cortices were identified on the basis of stereotaxic components during in vivo imaging. At the end of the imaging session, the location was further verified by injecting a tracer into the imaged area and fixing the brain. The injections were then identified in fixed sections and compared with the mouse atlas (supplemental Fig. $1 \mathrm{~A}$, available at www.jneurosci.org as supplemental material). In most cases, imaged areas were located in the primary sensory cortex, although occasionally, secondary sensory cortices were imaged. In some animals, imaging in primary cortex was confirmed by allowing the dye to transport for $2 \mathrm{~d}$ and observing labeling in the appropriate nucleus of the sensory thalamus (supplemental Fig. $1 B$, available at www.jneurosci.org as supplemental material).

Imaged spines in all sensory areas showed dynamic changes in shape and length as described previously in vivo and in vitro ( $\mathrm{Fi}$ scher et al., 1998; Dunaevsky et al., 1999; Lendvai et al., 2000; Majewska and Sur, 2003; Oray et al., 2004). The changes included elongation, retraction, spine shape change and displacement, and filopodial growth. Motile spines were frequently located next to stable spines, suggesting that spine motility is regulated on a spine-by-spine basis (Fig. 1).

\section{Spines are more dynamic in auditory and somatosensory} cortices than in visual cortex

Dendritic spines located on the apical tuft of layer 5 neurons in the visual cortex were the most stable of the protrusions studied $(0.0215 \pm 0.00050 \mu \mathrm{m} / \mathrm{min} ; n=261$ spines from 6 animals) (Fig. 2A). As observed previously (Majewska and Sur, 2003), at P28, most spines in this population show adult morphologies, with the majority being mushroom spines (62\%) and few filopodia (4\%) being observed (Fig. 2 B). Additionally, stubby and mushroom spines were most stable and abundant, whereas thin spines were significantly more motile ( $p<0.05$ ) but comprised $<10 \%$ of the spine population. Filopodia were rarely found but were always highly motile compared with spines $(p<0.001)$.

Spines located in the primary somatosensory cortex were significantly more motile at this age than those in visual cortex $(0.0250 \pm 0.0008 \mu \mathrm{m} / \mathrm{min} ; n=221$ spines from 4 animals; $p<$ 0.001 ) (Fig. 2A). Again, most spines in somatosensory cortex were adult-like mushroom spines (55\%), but more thin spines $(16 \%)$ and filopodia $(9 \%)$ were observed than in visual cortex (Holtmaat et al., 2005) (Fig. 2 B). Mushroom spines in somatosensory cortex were more motile than those in visual cortex (visual, $0.0205 \pm 0.0005 \mu \mathrm{m} / \mathrm{min}$; somatosensory, $0.0247 \pm 0.0008$ $\mu \mathrm{m} / \mathrm{min} ; p<0.001)$. There were no significant differences in the motility of stubby spines, thin spines, or filopodia $(p>0.1)$.

Interestingly, dendritic spines located in the auditory cortex were also significantly more motile than those in visual cortex $(0.0271 \pm 0.0010 \mu \mathrm{m} / \mathrm{min} ; n=236$ spines from 6 animals; $p<$ 0.001 ) (Fig. $2 A$ ). This effect was not caused by an overabundance of motile filopodia in the sample, because most protrusions exhibited spine-like morphologies (97\%, with 56\% classified as mushroom spines), and the incidence of filopodia was comparable with that observed in visual cortex and lower than that observed in somatosensory cortex (Fig. 2 B). Additionally, all spine classes (mushroom, stubby, and thin) showed increased motility $(p<0.05)$ compared with visual cortical spines, apart from filopodia, which were equally motile in all sensory cortices studied (Fig. 2C).

\section{Rewiring visual input into auditory cortex does not downregulate spine motility}

The differences observed between the motility of dendritic spines in different sensory cortices could depend on the nature of the sensory input or on factors intrinsic to the cortical area itself. To distinguish between these two possibilities, we imaged spine motility in the same spine population in animals in which retinal input had been routed into the auditory thalamus, providing visual drive to auditory cortex (Sharma et al., 2000; Lyckman et al., 2001). We reasoned that, if the nature of the sensory input were instructive to structural plasticity, then spines in auditory cortex of rewired mice would show increased stability when compared with wild-type mice. Spines in the auditory cortex of rewired mice, however, did not show increased stability $(0.0272 \pm$ $0.00057 \mu \mathrm{m} / \mathrm{min} ; n=281$ spines from 8 animals) (Fig. $2 A$ ). In fact, spine motility in auditory cortex of rewired mice was not statistically different from that in normal mice and was statistically elevated from that observed in visual cortex $(p<0.001)$, suggesting that the modality of sensory input alone is not enough to alter basal spine motility. 

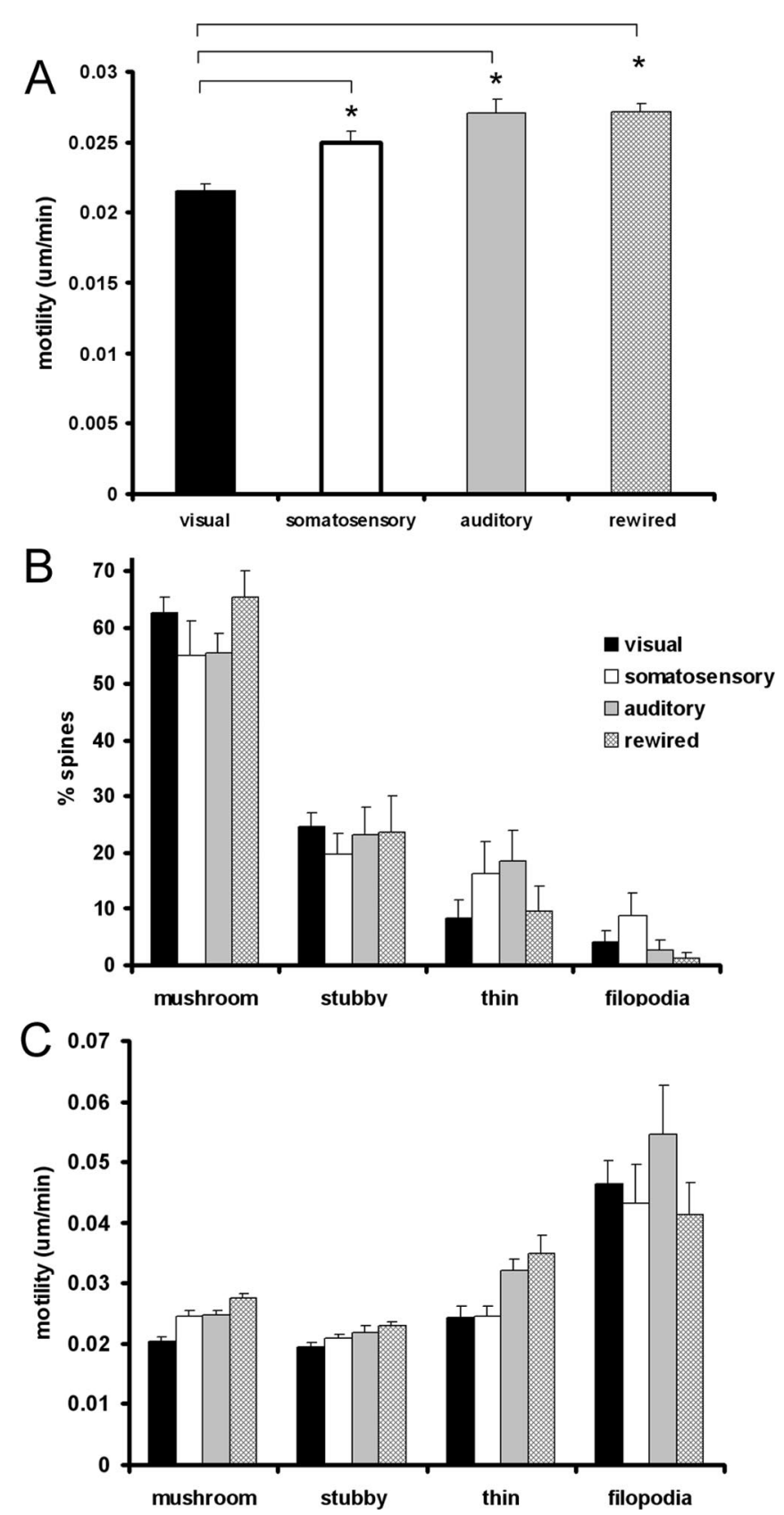

Figure 2. Spine motility is higher in auditory and somatosensory cortices than in visual cortex. $\boldsymbol{A}$, Graph showing spine motility in visual, somatosensory, and auditory cortices. Spine motility in auditory and somatosensory cortices is significantly higher $\left({ }^{*} p<0.001\right)$ than in visual cortex. Rewiring visual input into auditory cortex does not alter spine motility. $\boldsymbol{B}$, Graph showing dendritic spine class distributions in different cortical areas. In all areas at these ages, mushroom spines are most common, with a smaller proportion of thin and stubby spines. Filopodia are rare at these ages, although somatosensory cortex maintains a higher proportion of these immature protrusions ( 15\% of all protrusions). C, Spine motility of all spine classes is upregulated in auditory cortex compared with visual cortex $(p<0.05)$. The motility of mushroom spines is upregulated in somatosensory cortex compared with visual cortex $(p<0.001)$. Filopodia are highly motile in all regions studied.

\section{Imaging spine and axon terminal turnover in vivo}

To determine whether the differences observed in spine motility between different cortices at P28 translate to differences in the remodeling of cortical circuitry in the adult animal, we performed long-term imaging experiments of both postsynaptic dendritic spines and presynaptic axon terminals (Fig. 3) (see Fig. 5 ). In one set of animals, control images were taken at P40, and the same areas were then reimaged at P47. In another group of animals, control images were taken at P40 and then the same areas were reimaged at P61. Animals were imaged through thinned skull, and the scalp skin was sutured between imaging sessions. At the end of the last imaging session, tracer was injected into the imaged region and the imaged area was later identified on the basis of this injection in fixed sections (supplemental Fig. 1 , available at www.jneurosci.org as supplemental material).

Dendritic spines are equally persistent in visual, somatosensory, and auditory cortices

Previous studies showed contradictory results regarding spine persistence: one study yielded high turnover rates in somatosensory cortex (Trachtenberg et al., 2002), whereas another study showed highly stable spines in visual cortex (Grutzendler et al., 2002). Because we had already established differing rates of spine dynamics in different sensory areas in juvenile (P28) animals, we wondered whether the different sensory regions analyzed in the two studies could account for these discrepancies in turnover. To address this issue, we measured dendritic spine turnover in visual, somatosensory, and auditory cortices.

Turnover measurements were performed over a 1-3 week period in young adult animals (starting at P40) to aid in comparison with previous studies (Fig. 3). Cortical spines were remarkably stable over 1 week (stable spines, $89.0 \pm 5.4 \%$ of all protrusions; $n=1490$ in 13 animals) as well as over 3 weeks (stable spines, $84.4 \pm 9.7 \%$ of all protrusions; $n=1154$ in 15 animals). At these ages, spine loss $(13.4 \pm 7.1 \%)$ was greater than spine gain $(8.8 \pm$ $7.4 \% ; p<0.005)$. These data suggest that there is a small $(\sim 15 \%)$ subpopulation of synapses that remodel over a period of 1 week in young adult animals. The number of spines that turn over is, however, an overestimate given that any changes in imaging conditions might allow some spines to fall below or above the detection threshold on different imaging days.

Turnover rates were comparable in all three sensory areas studied (Fig. 3C). Approximately $90 \%$ of spines persisted over a period of 1 week (visual, $93.2 \pm 1.77 \%, n=282$ spines in 3 animals; somatosensory, $88.8 \pm 3.96 \%, n=345$ in 3 animals; auditory, $91.4 \pm 4.5 \%, n=286$ spines in 4 animals). Dendritic spines were also persistent over a period of 3 weeks in all sensory areas studied (Fig. $3 C$ ). Eighty to $90 \%$ of the same spines could be reidentified after a period of 3 weeks (visual, $78.2 \pm 12.1 \%, n=$ 450 spines in 6 animals; somatosensory, $87.5 \pm 5.4 \%, n=309$ spines in 3 animals; auditory, $88.5 \pm 7.3, n=290$ spines in 4 animals). This suggests that there is either a large population of spines that remains stable during early adulthood and a smaller population that turns over or that there is a limited amount of turnover after the animal is older than P40.

\section{Dendritic spine types and turnover}

At the ages studied (P28-P61), most spines had adult mushroom-like morphologies (supplemental Fig. 2, available at www.jneurosci.org as supplemental material). Between P28 and $\mathrm{P} 40$, there was a general decrease in the number of thin spines and filopodia in all sensory modalities (Zuo et al., 2005) and an increase in the number of stubby spines. After P40, there was little change in spine class composition, and the types of spines present did not differ significantly between the different sensory modalities studied $(p>0.05)$.

Mushroom spines were most common at these ages and were therefore responsible for both the stability and the limited turnover observed. Mushroom spines made up $\sim 60 \%$ of all stable protrusions and $\sim 40 \%$ of both lost and new protrusions (Fig. 
A

$\mathrm{P} 40$

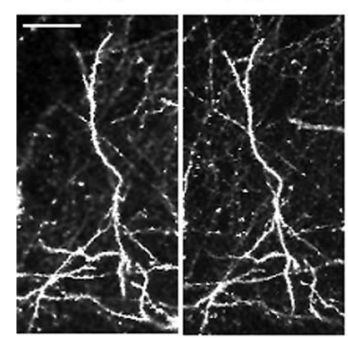

B

$\mathrm{P} 40$

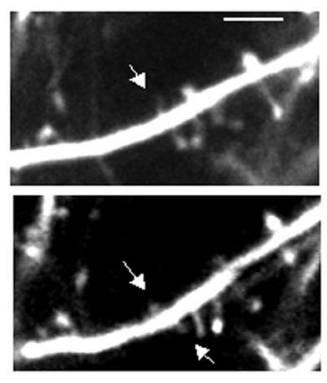

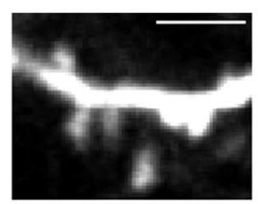

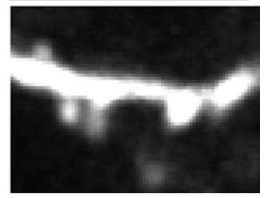

C

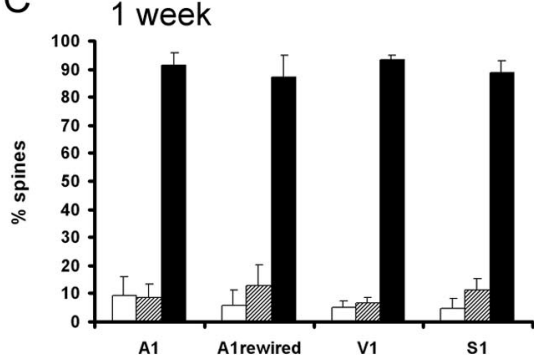

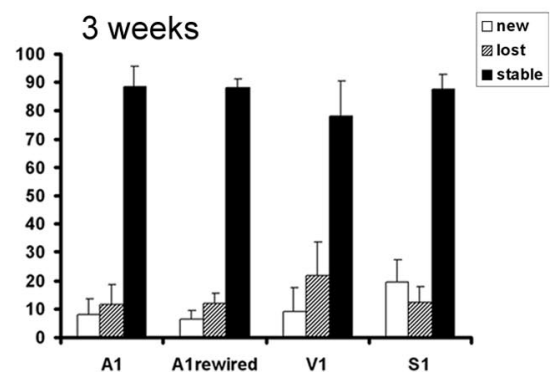

A

Figure 3. Low turnover rates of dendritic spines in young adult cortex. $\boldsymbol{A}$, In vivo images of layer 5 apical dendrites taken during chronic imaging experiments in auditory cortex. Images are projected in two dimensions from $50 Z$ sections taken $3 \mu \mathrm{m}$ apart. Scale bar, $50 \mu \mathrm{m}$. Left, First time point at P40. Right, Second time point at P61. B, In vivo images of dendritic structure taken during chronic imaging experiments in somatosensory (left) and visual (right) cortices. Images are projected in two dimensions from $Z$ sections taken $1 \mu \mathrm{m}$ apart. Scale bar, $5 \mu \mathrm{m}$. Notice that, after 3 weeks, the same dendritic spines can easily be identified. Arrows in the top panels mark spines that are lost, and arrows in the bottom panels mark spines that are formed de novo. C, Graphs showing turnover of dendritic spines in different cortical areas after a 1 week period (left) and after a 3 week period (right). Eighty to $90 \%$ of dendritic spines can be reidentified during the second imaging session under these conditions. No statistically significant differences in turnover can be observed between different sensory regions. A1, Auditory cortex; V1, visual cortex; S1, somatosensory cortex.

$4 A)$. Thin spines were less common $(\sim 15 \%$ of the total spine population) but were less likely to be stable than either mushroom or stubby spines. A total of $90 \%$ of all mushroom and stubby spines were stable over 3 weeks, whereas only $72 \%$ of thin spines persisted during this time (Fig. $4 B$ ), suggesting that thin spines may comprise a transient population that is susceptible to remodeling (Holtmaat et al., 2005). We cannot rule out that some of the thin spines we observed were filopodia in the process of extension or retraction.

To determine whether changes in spine shape occurred in stable synapses in early adulthood, we classified spines based on morphology in the first and second imaging sessions. We found that most spine types, excluding filopodia, maintained their classification throughout the imaging interval. Thus, $>50 \%$ of all mushroom, thin, and stubby spines could be reidentified with similar morphologies 1 or 3 weeks later (Fig. 4C, Table 1). Mushroom spines were least likely to change morphology, however, with $>80 \%$ maintaining a mushroom-like structure over 3 weeks. Thin spines were significantly more likely to undergo morphological changes ( $p<0.005$ ), with only $55 \%$ maintaining a thin-like structure over 3 weeks, again suggesting that thin spines are more susceptible to remodeling. Additionally, more remodeling was observed in the 3 versus 1 week imaging period (Fig. 4C) in all spine types, suggesting that morphological changes are ongoing in the cortex and may reflect activitydependent plasticity. This type of analysis captures only large changes in spine morphology, and it is likely that more subtle changes in spine size are more prevalent. These data were pooled from all cortical areas, and no significant differences were observed between visual, auditory, and somatosensory cortices.
Axon terminals are persistent in visual, somatosensory, and auditory cortices To determine the stability of presynaptic structures, we investigated the rates of axon terminal turnover in the same images used for dendritic spine turnover analysis. Axon terminals were identified on the basis of their morphology, as thick round structures or finger, spine-like protrusions on axons (Fig. $5 A, B$ ). In fixed sections, such structures colocalized with synapsin staining, indicating that they are likely to be functional presynaptic sites (supplemental Fig. $1 C$, available at www.jneurosci. org as supplemental material). Axon terminals were highly stable in all cortices studied (Fig. 5C). After a 1 week period, $>90 \%$ of axon terminals could be reidentified (visual, $90.0 \pm 0.8 \%, n=258$ in 3 animals; somatosensory, $92.9 \pm 3.4 \%, n=$ 231 in 3 animals; auditory, $94.9 \pm 3.3 \%$, $n=220$ in 4 animals).

In a 3 week imaging period, axon terminals were also persistent, with $80-90 \%$ of the same terminals being present in both imaging sessions (visual, $88.3 \pm$ $5.44 \%, n=425$ in 6 animals; somatosensory, $91.8 \pm 3.5 \%, n=133$ in 3 animals; auditory, $94.8 \pm 3.9 \%, n=236$ in 4 animals) (Fig. 5C). Again, rates of terminal turnover were low in 3 weeks of imaging, indicating that either a small population of terminals turns over or that turnover becomes less prevalent after P40. Terminals in the visual cortex turned over more than those in auditory cortex $(p<0.05)$. Also, rates of terminal loss and gain were well matched in all cortices during the 3 week imaging interval.

To understand whether changes in cortical circuitry occur by coordinated turnover of both presynaptic and postsynaptic elements, we compared dendritic spine and axon terminal turnover rates in our images. On average, at these ages, axon terminals were more stable than dendritic spines $(p<0.001)$ (Fig. $6 A, B)$. This was true for spines in visual, auditory, and somatosensory cortices. In all areas, the increased turnover of dendritic spines was primarily attributable to an increased rate of spine loss compared with that of axon terminals (Fig. $6 A, C$ ).

\section{Rewiring visual input into the auditory cortex does not affect} synaptic persistence

We investigated whether changing the type of sensory input to a cortical area would affect spine and axon terminal turnover by rewiring visual projections into the auditory system and investigating spine and terminal persistence in auditory cortex. This manipulation did not affect basal spine turnover rates in auditory cortex in either the 1 or 3 week imaging period (rewired cortex at 1 week, $87.3 \pm 7.6 \%, n=577$ in 4 animals; 3 weeks, $87.9 \pm 3.5 \%$, $n=253$ in 3 animals) (Fig. $3 B$ ). Spines were more likely to disappear ( 1 week, $6.8 \pm 1.8 \%$; 3 weeks, $12.1 \pm 3.5 \%$ ) than to appear ( 1 week, $5.1 \pm 2.1 \%$; 3 weeks, $6.5 \pm 2.8 \%$ ). Axon terminals were equally stable in rewired and nonrewired auditory cortex and turned over less than those in visual cortex $(p<0.05)$. Similar to the other sensory regions studied, terminals were more stable than dendritic spines (rewired cortex at 1 week, $93.8 \pm 3.8 \%, n=$ 
1 week

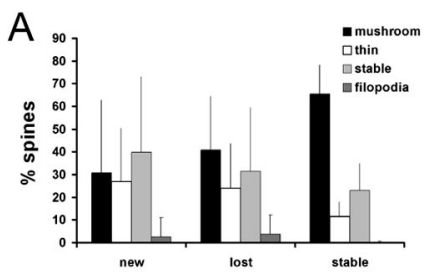

B

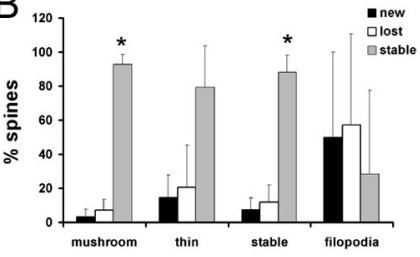

C

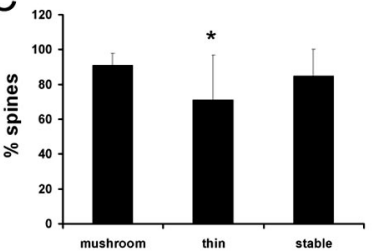

3 weeks
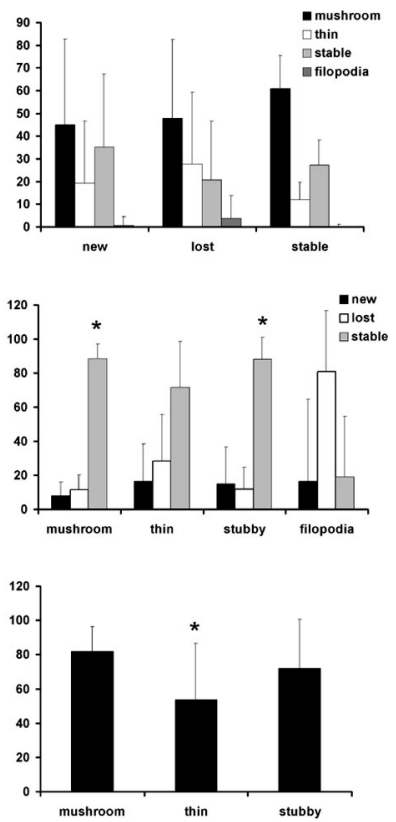

Figure 4. Thin spines are less stable than mushroom and stubby spines. $\boldsymbol{A}$, Mushroom spines make up the majority of stable spines in both the 1 week (left) and 3 week (right) imaging periods ( $p<0.001$ ). Thin spines are least likely to be stable over this imaging interval ( $p<$ $0.001)$. Data presented are the percentage of each spine class that comprise all new, lost, and stable spines (e.g., the number of mushroom spines that are stable normalized to the number of all stable protrusions observed). $\boldsymbol{B}$, Mushroom and stubby spines observed on the first day of imaging are most likely to be reidentified in the second imaging session (left, 1 week; right, 3 weeks). Thin spines are more likely to be lost and also to be formed than either mushroom or stubby spines $\left({ }^{*} p<0.05\right)$. Very few filopodia remain stable during the imaging interval. Data presented are the percentage of new, lost, and gained spines in each spine class. C, Morphological changes occur in stable spines. Graph showing the percentage of stable spines of each spine class that maintain their morphological classification at 1 week (left) and 3 week (right) imaging intervals. The majority (80-90\%) of mushroom spines maintain mushroom-like morphologies over time, whereas significantly fewer $\left(50-70 \%\right.$; $\left.{ }^{*} p<0.005\right)$ thin spines remain thin. Notice that fewer spines in all classes maintain their morphological classification over the 3 week than the 1 week imaging interval.

415 in 4 animals; 3 weeks, $95.3 \pm 3.7 \%, n=219$ in 3 animals) (Fig. 5B).

\section{Dendritic spine stability is likely to be a general feature of cortical circuitry}

Our results so far have been limited to synapses occurring on the apical tuft of layer 5 neurons in sensory cortices in young adult animals. To determine whether persistence of dendritic spines is limited to this subset of synapses or whether it is a more general feature of cortical synapses, we imaged dendritic spines of layer 5 neurons in the motor cortex of one P40 animal. After 3 weeks, $\sim 80 \%$ of dendritic spines were stable in this animal, suggesting that dendritic spine stability might not be an exclusive feature of sensory cortex (stable spines, $87.5 \%$; new spines, $12.5 \%$; lost spines, $18.9 \% ; n=71$ spines).

Additionally, in some cases, layer $2 / 3$ neurons in somatosensory cortex were also labeled. We imaged dendritic spines on the proximal apical dendrite of these neurons up to $300 \mu \mathrm{m}$ below the surface of the pia. Over a 1 week period, $90 \%$ of spines were stable with more spines being lost than being formed de novo (stable spines, $90.6 \pm 3.7 \%$; new spines, $3.3 \pm 3.4 \%$; lost spines, $9.4 \pm 3.7 \% ; n=187$ spines from 2 animals) (Fig. 7). Additionally, axon terminals were also very stable (stable, $94.5 \pm 2.6 \%$; new,
$2.8 \pm 3.3 \%$; lost, $5.5 \pm 2.6 \% ; n=137$ from 2 animals). These rates of spine formation and disassembly are comparable with those seen on the apical tuft of layer 5 neurons in visual, somatosensory, and auditory cortices (see above). The data from layer $2 / 3$ and from layer 5 neurons in motor cortex suggest that it is likely that stability of presynaptic and postsynaptic structures in adult animals is a general property of cortical pyramidal neurons.

\section{Discussion}

We show that rapid morphological alterations in dendritic spines on the same neuron type (layer 5 pyramid), on the same parts of the dendritic tree (the apical tuft), are a function of cortical area, with dynamics being more pronounced in auditory and somatosensory cortex compared with visual cortex at the same developmental stage. In adult animals, however, rates of synaptic turnover are low and comparable between the cortical regions studied, with terminals being more stable than spines. Interestingly, rewiring visual input into the auditory cortex does not alter spine dynamics, suggesting that basal synaptic rearrangements may be intrinsic to the cortical area. Our results suggest that, in young and mature animals, cortical plasticity may be mediated by changes in synaptic morphology with limited assembly and disassembly of synaptic structures.

\section{Activity dependence of spine dynamics}

Morphological changes in spines may be involved in the formation of new connections during synaptogenesis and network remodeling (Bonhoeffer and Yuste, 2002). Our experiments suggest that, in young animals, spines turn over infrequently but undergo morphological changes. Thin spines are the most motile spines at P28 and are the most likely to turn over and change morphology at $\mathrm{P} 40$. This supports the view that thin spines carry weak synapses (Matsuzaki et al., 2001) and, when potentiated, grow to become mushroom spines (Matsuzaki et al., 2004; Okamoto et al., 2004). Thin spines have been implicated previously as preferential sites of synaptic remodeling (Holtmaat et al., 2005), whereas large mushroom spines may be resistant to both potentiation and additional increases in size (Matsuzaki et al., 2004). In our study, we did not see preferential transitions between spine classes, suggesting that neck length, neck diameter, and head diameter can be regulated independently. These parameters may be fine tuned by activity allowing spines to regulate synaptic calcium increases (Majewska et al., 2000a; Noguchi et al., 2005; Oertner and Matus, 2005).

Differences in spine dynamics between sensory cortical areas Differences in basal spine motility between sensory areas suggest that, in vivo, spine motility is driven by sensory input. Our rewiring experiments, however, suggest that this is unlikely. In rewired animals, the auditory cortex experiences patterns of activity driven by visual input commencing shortly after birth. Synaptic dynamics in these animals, however, are not affected by this different activity pattern. There are several explanations compatible with this result. First, activity may be important during the critical period, which closes at approximately P28 in auditory cortex (Zhang et al., 2001). Similarly, activity-dependent effects on spine motility in somatosensory (Lendvai et al., 2000) and visual (Majewska and Sur, 2003; Oray et al., 2004) cortices decline by P14 and P28, respectively. Second, effects on spine motility were observed after gross manipulation of activity in vitro (Fischer et al., 2000; Korkotian and Segal, 2001) or deprivation paradigms in vivo (Lendvai et al., 2000; Majewska and Sur, 2003; Oray et al., 2004), suggesting that overall levels of activity may be more im- 
Table 1. Spine morphology transitions

\begin{tabular}{llllll}
\hline & To mushroom & To thin & To stubby & To filopodia & \% Total protrusions \\
\hline Mushroom & $59.7 \%(50.4 \%)$ & $2.4 \%(4.1 \%)$ & $3.2 \%(6.2 \%)$ & $0(0.1 \%)$ & $60.4 \%$ \\
Thin & $2.6 \%(3.3 \%)$ & $8 \%(6.5 \%)$ & $1.1 \%(2.1 \%)$ & $0.1 \%(0 \%)$ & $13.9 \%$ \\
Stubby & $3 \%(4.6 \%)$ & $0.6 \%(2.3 \%)$ & $19 \%(20.1 \%)$ & $0 \%(0 \%)$ & $24.8 \%$ \\
Filopodia & $0.2 \%(0 \%)$ & $0.02 \%(0.2 \%)$ & $0.04 \%(0 \%)$ & $0 \%(0 \%)$ & $0.9 \%$ \\
\hline
\end{tabular}

Transitions occurring between different spine types as a percentage of all stable spines observed during the imaging period. Values given are for a 1 week observation period, with values for 3 weeks shown in parentheses. All protrusions were most likely to maintain their classification over time except for filopodia, which occasionally converted to spines. The types of observed transitions were similar in the 1 and 3 week observation periods.
A

P40

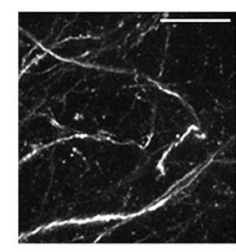

C

1 week

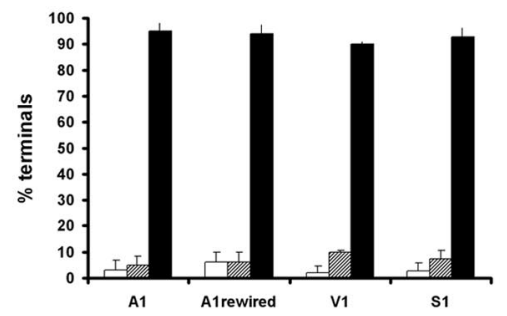

B

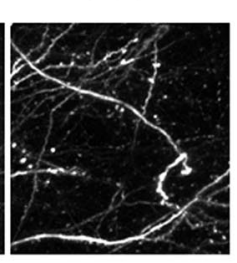

P61

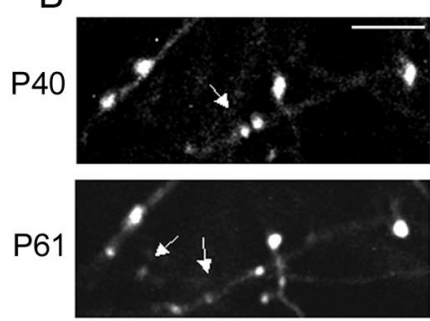

3 weeks
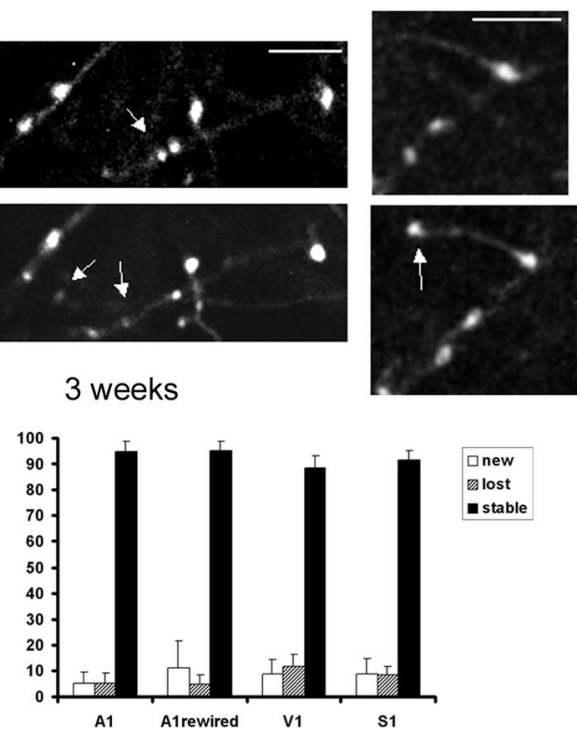

Figure 5. Low turnover rates of axon terminals in young adult cortex. $\boldsymbol{A}$, In vivo images of layer 5 apical dendrites taken during chronic imaging experiments in visual cortex. Images are projected in two dimensions from $50 Z$ sections taken $3 \mu \mathrm{m}$ apart. Scale bar, $50 \mu \mathrm{m}$. Left, First time point at P40. Right, Second time point at P61. B, In vivo images of axonal structure taken during chronic imaging experiments in somatosensory (left) and visual (right) cortices. Images are projected in two dimensions from Z sections taken $1 \mu \mathrm{m}$ apart. Scale bar, $5 \mu \mathrm{m}$. Notice that, after 3 weeks, the same axon terminals can easily be identified. Arrows in the top panels mark terminals that are lost, and arrows in the bottom panels mark terminals that are formed de novo. C, Graphs showing turnover of axon terminals in different cortical regions after a 1 week period (left) and after a 3 week period (right panel). Ninety to $95 \%$ of terminals can be reidentified during the second imaging session under these conditions. Axon terminals were very stable in all sensory areas studied. A1, Auditory cortex; V1, visual cortex; S1, somatosensory cortex.
However, these differences are not apparent when examining spine turnover in young adult animals, and, at 6-9 weeks, spine class composition is similar in the two areas. Our data support the view that spines in visual and somatosensory cortices are stable ( $\sim 85 \%$ over 3 weeks starting at P40). At these ages, we also find higher rates of spine elimination than addition, in agreement with Zuo et al. (2005). Additionally, our data suggest that spine stability may be a general feature of cortical neurons.

Although surgical preparation could be responsible for the difference in turnover in these studies, recent experiments show that comparable images are obtained with the window (Trachtenberg et al., 2002; Holtmaat et al., 2005) and thinned skull (Grutzendler et al., 2002; Zuo et al., 2005) preparations. Our control experiments also showed comparable results in the two preparations (see Materials and Methods). Additionally, differences in spine motility were observed in thinned skull preparations at P28, and thin spines were easily found in somatosensory cortex at these ages. It is possible that other factors affect spine turnover. For instance, the frequency of imaging could cause photodamage or increase the delivery of anesthetics that affect cortical function and spine morphology (Kaech et al., 1999). Additionally, there might be differences caused by environmental and rearing conditions (Cancedda et al., 2004).

Although our data point to spines being more stable than originally reported in somatosensory cortex (Trachtenberg et al., 2002), it is necessary to note that, over 3 portant than its fine structure. Third, rewiring may affect too few synapses to be detected with our imaging paradigm. This is unlikely because retinal projections to the auditory thalamus are extensive, spanning the entire ventral division of the MGN (Ellsworth et al., 2005). Furthermore, rewiring elicits functional changes in the network architecture of auditory cortex and can drive visual responses and behavior (Sharma et al., 2000; von Melchner et al., 2000; Newton et al., 2004). We favor the fourth possibility, that cues intrinsic to the cortical area regulate synaptic dynamics.

\section{Dendritic spines are persistent in adult cortex}

Previous studies yielded contradictory results on dendritic spine turnover in young adult cortex. In somatosensory cortex, $60 \%$ of spines remained stable over $8 \mathrm{~d}$ (Trachtenberg et al., 2002). In visual cortex, however, $90 \%$ of spines persisted over a period of months (Grutzendler et al., 2002). Recent reports agree that, in older animals (>3 months) and longer imaging intervals, spines are equally stable in both cortices (Holtmaat et al., 2005; Zuo et al., 2005). Our data in juvenile animals confirm that spines in somatosensory cortex are more motile than those in visual cortex. Additionally, in developing animals, thin spines and filopodia are more prevalent in somatosensory cortex (Holtmaat et al., 2005). weeks, $10-20 \%$ of spines are lost and $\sim 10 \%$ are formed. Although some of this turnover is possibly explained by limitations of the imaging technique in identifying functional synapses, it is likely that the adult cortex maintains a level of structural plasticity that is greater than previously expected. It is likely that the remodeling of synapses made onto inhibitory neurons is governed by different rules as dendrites of these cells are structrally more plastic than those on pyramidal neurons (Lee et al., 2006). Thus, although the adult cortex learns and adapts through altering the strength of existing synapses, it likely also remodels through physically disconnecting and wiring up new synapses. Such changes in connectivity must be tightly regulated so as not to affect long-term memory but allow flexibility during learning of new behaviors. The fact that adult synapses are structurally labile may prove important for the treatment of brain trauma and agerelated cognitive defects.

\section{Spines are more plastic than axon terminals}

Although spine turnover rates have been studied, little is known about the dynamics of axonal structures (but see Portera-Cailliau et al., 2005). Our data suggest that axon terminals are highly stable, confirming previous reports on synaptic motility in the cerebellum (Deng and Dunaevsky, 2005). Because higher actin 
A

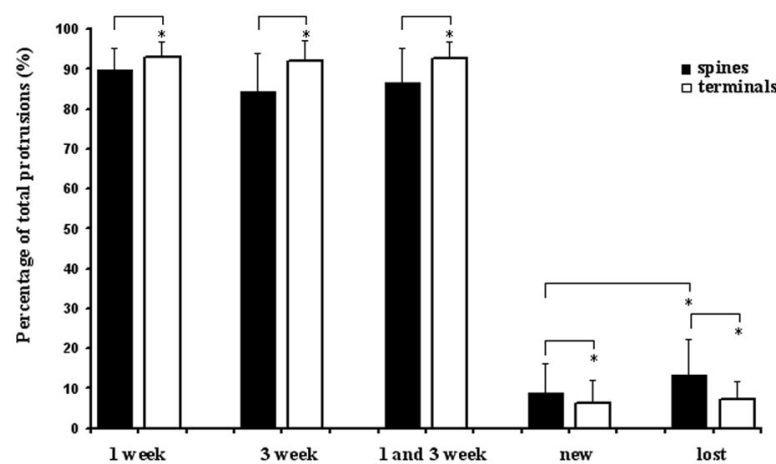

$\mathrm{B}$
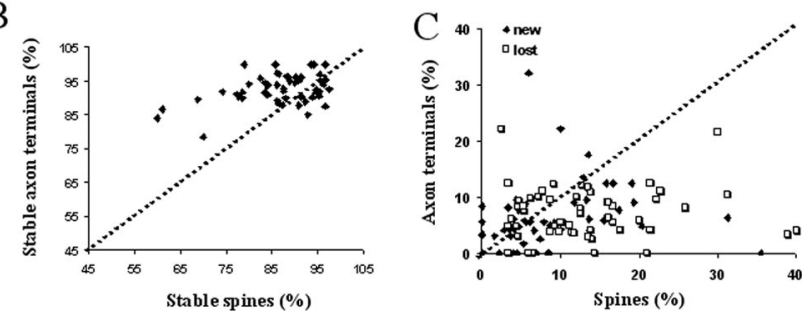

Figure 6. Dendritic spines show higher rates of turnover than axon terminals. $\boldsymbol{A}$, Dendritic spines were more stable then axon terminals at both 1 and 3 week imaging intervals. Spines were more likely to be lost during the imaging period, and rates of loss were higher for dendritic spines than axon terminals. ${ }^{*} p<0.05$. $\boldsymbol{B}$, Graph plotting the percentage of stable terminals versus stable spines in individual imaged areas from different animals. Notice that axon terminals are more likely to be stable than nearby dendritic spines. C, Graph plotting the percentage of new (black diamonds) and lost (white squares) axon terminals in each imaged region versus the percentage of new and lost dendritic spines in the same region. Dendritic spines are more likely to be lost during the imaging session than axon terminals.

concentrations are generally associated with motile structures, it is perhaps not surprising that the presynaptic terminal with less actin is more stable than the actin-filled postsynaptic spine (Matus et al., 1982). The difference in turnover rates is primarily mediated by a lower rate of terminal loss in the young adult animal. This suggests that dendritic spines are overproduced during synaptogenesis and that pruning of spurious synapses in the mature animal progresses through the elimination of excess dendritic spines.

At least three technical aspects must be considered in these data. First, boutons are larger than spines and are therefore easier to detect in vivo. It is unlikely, however, that the differences observed are attributable to detection thresholds, because axons, which are much thinner and dimmer than most spines, were always visible in subsequent imaging sessions. Additionally, finger-like terminals were also more persistent than dendritic spines (data not shown). Second, although spine morphology has routinely been used to identify postsynaptic sites, boutons determined morphologically may not correspond to functional presynaptic sites (Shepherd and Harris, 1998). Unless our morphological criteria do not pick up a population of transient presynapses, this would, however, lead to an underestimation of the stability of terminals. Third, we cannot be sure that the bouton population we are studying makes synapses with the spines imaged. Although in some cases we see close apposition of these two elements suggesting that connections between them are possible, determining the connectivity would require an enormous effort and is beyond the scope of this study.

Our results suggest that dendritic spines are persistent in sensory cortices and, possibly, in all of cortex. A small population of spines, however, turns over even in adult cortex, possibly allow-
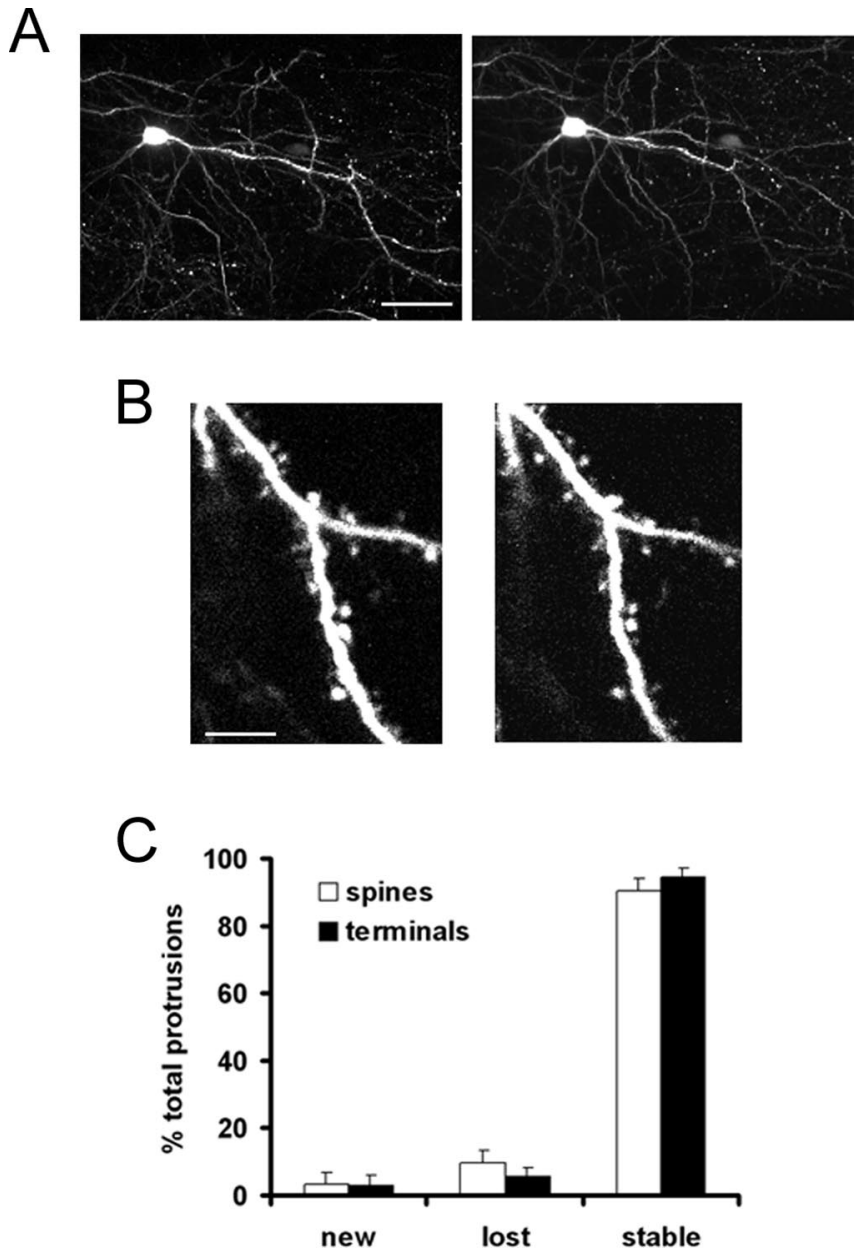

Figure 7. Dendritic spine turnover in layer $2 / 3$ neurons. $\boldsymbol{A}$, In vivo images of a layer $2 / 3$ neuron in somatosensory cortex taken 1 week apart. Images are collapsed from $Z$ sections taken $3 \mu \mathrm{m}$ apart. Scale bar, $50 \mu \mathrm{m}$. B, Dendritic sections from neuron in $\boldsymbol{A}$. Notice that most of the same spines can be reidentified after a 1-week-long period. Images are collapsed from Z sections taken $1 \mu \mathrm{m}$ apart. Scale bar, $5 \mu \mathrm{m}$. C, Graph showing percentages of stable, new, and lost spines. More than $90 \%$ of spines can be reidentified over a 1 -week-long period, suggesting that persistence may be a general property of cortical spines located on pyramidal neurons.

ing for pruning and rearrangements of synapses. This scenario is appealing in that it allows the setting up of a coarse scaffold of connectivity during development that can be refined through changes in synaptic strength but not easily dismantled or rearranged in the adult animal (Filbin, 2003). In fact, presynaptic elements appear more structurally stable than dendritic spines, suggesting that they act as a scaffold for connectivity, whereas spines carry most of the structural plasticity in adult synapses. Indeed, our data show that, although spines are persistent in the adult animal, they still undergo changes in structure, suggesting that they retain a level of structural plasticity that might correspond to changes in synaptic strength.

\section{References}

Bonhoeffer T, Yuste R (2002) Spine motility. Phenomenology, mechanisms, and function. Neuron 35:1019-1027.

Cancedda L, Putignano E, Sale A, Viegi A, Berardi N, Maffei L (2004) Acceleration of visual system development by environmental enrichment. J Neurosci 24:4840-4848.

Deng J, Dunaevsky A (2005) Dynamics of dendritic spines and their afferent terminals: spines are more motile than presynaptic boutons. Dev Biol 2777:366-377.

DeRobertis EDP, Bennett H (1955) Some features of the submicroscopic 
morphology of synapses in frog and earthworm. J Biophys Biochem Cytol $1: 47-58$.

Dunaevsky A, Tashiro A, Majewska A, Mason C, Yuste R (1999) Developmental regulation of spine motility in the mammalian central nervous system. Proc Natl Acad Sci USA 96:13438-13443.

Ellsworth CA, Lyckman AW, Feldheim DA, Flanagan JG, Sur M (2005) Ephrin-A2 and -A5 influence patterning of normal and novel retinal projections to the thalamus: conserved mapping mechanisms in visual and auditory thalamic targets. J Comp Neurol 488:140-151.

Engert F, Bonhoeffer T (1999) Dendritic spine changes associated with hippocampal long-term synaptic plasticity. Nature 399:66-70.

Feng G, Mellor RH, Bernstein M, Keller-Peck C, Nguyen QT, Wallace M, Nerbonne JM, Lichtman JW, Sanes JR (2000) Imaging neuronal subsets in transgenic mice expressing multiple spectral variants of GFP. Neuron 28:41-51.

Filbin MT (2003) Myelin-associated inhibitors of axonal regeneration in the adult mammalian CNS. Nat Rev Neurosci 4:703-713.

Fischer M, Kaech S, Knutti D, Matus A (1998) Rapid actin-based plasticity in dendritic spines. Neuron 20:847-854.

Fischer M, Kaech S, Wagner U, Brinkhaus H, Matus A (2000) Glutamate receptors regulate actin-based plasticity in dendritic spines. Nat Neurosci 3:887-894.

Gray EG (1959) Electron microscopy of synaptic contacts on dendrite spines of the cerebral cortex. Nature 183:1592-1593.

Grutzendler J, Kasthuri N, Gan WB (2002) Long-term dendritic spine stability in the adult cortex. Nature 420:812-816.

Harris KM, Jensen FE, Tsao B (1992) Three-dimensional structure of dendritic spines and synapses in rat hippocampus (CA1) at postnatal day 15 and adult ages: implications for the maturation of synaptic physiology and long-term potentiation. J Neurosci 12:2685-2705.

Harris KM, Fiala JC, Ostroff L (2003) Structural changes at dendritic spine synapses during long-term potentiation. Philos Trans R Soc Lond B Biol Sci 358:745-748.

Hayashi Y, Majewska A (2005) Dendritic spine geometry: functional implication and regulation. Neuron 46:529-532.

Heynen AJ, Quinlan EM, Bae DC, Bear MF (2000) Bidirectional, activitydependent regulation of glutamate receptors in the adult hippocampus in vivo. Neuron 28:527-536.

Holtmaat AJ, Trachtenberg JT, Wilbrecht L, Shepherd GM, Zhang X, Knott GW, Svoboda K (2005) Transient and persistent dendritic spines in the neocortex in vivo. Neuron 45:279-291.

Kaech S, Brinkhaus H, Matus A (1999) Volatile anesthetics block actin-based motility in dendritic spines. Proc Natl Acad Sci USA 96:10433-10437.

Koester HJ, Sakmann B (1998) Calcium dynamics in single spines during coincident pre- and postsynaptic activity depend on relative timing of back-propagating action potentials and subthreshold excitatory postsynaptic potentials. Proc Natl Acad Sci USA 95:9596-9601.

Konur S, Yuste R (2004) Developmental regulation of spine and filopodial motility in primary visual cortex: reduced effects of activity and sensory deprivation. J Neurobiol 59:236-246.

Korkotian E, Segal M (2001) Regulation of dendritic spine motility in cultured hippocampal neurons. J Neurosci 21:6115-6124.

Lang C, Barco A, Zablow L, Kandel ER, Siegelbaum SA, Zakharenko SS (2004) Transient expansion of synaptically connected dendritic spines upon induction of hippocampal long-term potentiation. Proc Natl Acad Sci USA 101:16665-16670.

Lee WC, Huang H, Feng G, Sanes JR, Brown EN, So PT, Nedivi E (2006) Dynamic remodeling of dendritic arbors in GABAergic interneurons of adult visual cortex. PLos Biol 4:e29.

Lendvai B, Stern A, Chen B, Svoboda K (2000) Experience-dependent plasticity of dendritic spines in the developing rat barrel cortex in vivo. Nature 404:876-881.

Liu L, Wong TP, Pozza MF, Lingenhoehl K, Wang Y, Sheng M, Auberson YP, Wang YT (2004) Role of NMDA receptor subtypes in governing the direction of hippocampal synaptic plasticity. Science 304:1021-1024.

Lyckman AW, Jhaveri S, Feldheim DA, Vanderhaeghen P, Flanagan JG, Sur M (2001) Enhanced plasticity of retinothalamic projections in an ephrinA2/A5 double mutant. J Neurosci 21:7684-7690.

Majewska A, Sur M (2003) Motility of dendritic spines in visual cortex in vivo: changes during the critical period and effects of visual deprivation. Proc Natl Acad Sci USA 100:16024-16029.
Majewska A, Tashiro A, Yuste R (2000a) Regulation of spine calcium dynamics by rapid spine motility. J Neurosci 20:8262-8268.

Majewska A, Yiu G, Yuste R (2000b) A custom-made two-photon microscope and deconvolution system. Pflügers Arch 441:398-408.

Maletic-Savatic M, Malinow R, Svoboda K (1999) Rapid dendritic morphogenesis in CA1 hippocampal dendrites induced by synaptic activity. Science 283:1923-1927.

Matsuzaki M, Ellis-Davies GC, Nemoto T, Miyashita Y, Iino M, Kasai H (2001) Dendritic spine geometry is critical for AMPA receptor expression in hippocampal CA1 pyramidal neurons. Nat Neurosci 4:1086-1092.

Matsuzaki M, Honkura N, Ellis-Davies GC, Kasai H (2004) Structural basis of long-term potentiation in single dendritic spines. Nature 429:761-766.

Matus A (2005) Growth of dendritic spines: a continuing story. Curr Opin Neurobiol 15:67-72.

Matus A, Ackermann M, Pehling G, Byers HR, Fujiwara K (1982) High actin concentrations in brain dendritic spines and postsynaptic densities. Proc Natl Acad Sci USA 79:7590-7594.

Nagerl UV, Eberhorn N, Cambridge SB, Bonhoeffer T (2004) Bidirectional activity-dependent morphological plasticity in hippocampal neurons. Neuron 44:759-767.

Newton JR, Ellsworth C, Miyakawa T, Tonegawa S, Sur M (2004) Acceleration of visually cued conditioned fear through the auditory pathway. Nat Neurosci 7:968-973.

Noguchi J, Matsuzaki M, Ellis-Davies GCR, Kasai H (2005) Spine-neck geometry determines NMDA receptor-dependent calcium signaling in dendrites. Neuron 46:609-622.

Oertner TG, Matus A (2005) Calcium regulation of actin dynamics in dendritic spines. Cell Calcium 37:477-482.

Okamoto K, Nagai T, Miyawaki A, Hayashi Y (2004) Rapid and persistent modulation of actin dynamics regulates postsynaptic reorganization underlying bidirectional plasticity. Nat Neurosci 7:1104-1112.

Oray S, Majewska A, Sur M (2004) Dendritic spine dynamics are regulated by monocular deprivation and extracellular matrix degradation. Neuron 44:1021-1030.

Palay S (1965) Synapses in the central nervous system. J Biophys Biochem Cytol 2:193-201.

Portera-Cailliau C, Weimer RM, De Paola V, Caroni P, Svoboda K (2005) Diverse modes of axon elaboration in the developing neocortex. PLoS Biol 3:e272.

Ramón y Cajal S (1904) La textura del sistema nerviosa del hombre y los vertebrados. Madrid: Moya.

Sharma J, Angelucci A, Sur M (2000) Induction of visual orientation modules in auditory cortex. Nature 404:841-847.

Shepherd GM, Harris KM (1998) Three-dimensional structure and composition of CA3 $\rightarrow$ CA1 axons in rat hippocampal slices: implications for presynaptic connectivity and compartmentalization. J Neurosci 18:8300-8310.

Trachtenberg JT, Chen BE, Knott GW, Feng G, Sanes JR, Welker E, Svoboda $\mathrm{K}$ (2002) Long-term in vivo imaging of experience-dependent synaptic plasticity in adult cortex. Nature 420:788-794.

von Melchner L, Pallas SL, Sur M (2000) Visual behaviour mediated by retinal projections directed to the auditory pathway. Nature 404:871-876.

Yuan F, Chen Y, Dellian M, Safabakhsh N, Ferrara N, Jain RK (1996) Timedependent vascular regression and permeability changes in established human tumor xenografts induced by an anti-vascular endothelial growth factor/vascular permeability factor antibody. Proc Natl Acad Sci USA 93:14765-14770.

Yuste R, Denk W (1995) Dendritic spines as basic functional units of neuronal integration. Nature 375:682-684.

Yuste R, Majewska A, Holthoff K (2000) From form to function: calcium compartmentalization in dendritic spines. Nat Neurosci 3:653-659.

Zhang LI, Bao S, Merzenich MM (2001) Persistent and specific influences of early acoustic environments on primary auditory cortex. Nat Neurosci 4:1123-1130.

Zhou Q, Homma KJ, Poo MM (2004) Shrinkage of dendritic spines associated with long-term depression of hippocampal synapses. Neuron 44:749-757.

Zuo Y, Lin A, Chang P, Gan WB (2005) Development of long-term dendritic spine stability in diverse regions of cerebral cortex. Neuron 46:181189. 